\title{
Multiscale Petrov-Galerkin Method for High-Frequency Heterogeneous Helmholtz Equations
}

\author{
Donald L. Brown, Dietmar Gallistl, and Daniel Peterseim
}

\begin{abstract}
This paper presents a multiscale Petrov-Galerkin finite element method for time-harmonic acoustic scattering problems with heterogeneous coefficients in the high-frequency regime. We show that the method is pollutionfree also in the case of heterogeneous media provided that the stability bound of the continuous problem grows at most polynomially with the wave number $k$. By generalizing classical estimates of [Melenk, Ph.D. Thesis 1995] and [Hetmaniuk, Commun. Math. Sci. 5 (2007)] for homogeneous medium, we show that this assumptions of polynomially wave number growth holds true for a particular class of heterogeneous material coefficients. Further, we present numerical examples to verify our stability estimates and implement an example in the wider class of discontinuous coefficients to show computational applicability beyond our limited class of coefficients.
\end{abstract}

Acknowledgements The authors acknowledge the support given by the Hausdorff Center for Mathematics Bonn. D. Peterseim is supported by Deutsche Forschungsgemeinschaft in the Priority Program 1748 "Reliable simulation techniques in solid mechanics. Development of non-standard discretization methods, mechanical and mathematical analysis" under the project "Adaptive isogeometric modeling of propagating strong discontinuities in heterogeneous materials".

Donald L. Brown

School of Mathematical Sciences, The University of Nottingham, University Park, Nottingham, United Kingdom, e-mail: donald.brown(at)nottingham.ac.uk

Dietmar Gallistl

Institut für Numerische Simulation, Universität Bonn, Wegelerstr. 6, 53115 Bonn, Germany, e-mail: gallistl(at)ins.uni-bonn.de

Daniel Peterseim

Institut für Numerische Simulation, Universität Bonn, Wegelerstr. 6, 53115 Bonn, Germany, e-mail: peterseim(at)ins.uni-bonn.de 


\section{Introduction}

The time-harmonic acoustic wave-propagation is customarily described by the Helmholtz equation, which is of second-order, elliptic, but indefinite. Its numerical solution therefore exhibits severe difficulties especially in the regime of high wave numbers $k$. It is well-known that the mesh size $h$ required for the stability of a standard finite element method must be much smaller than a mesh size $H$ which would be sufficient for a reasonable representation of the solution. The phenomenon that the ratio $H / h$ tends to infinity as $k$ grows, is known as the pollution effect [1]. A method is referred to as pollution-free, if $h$ and $H$ have the same order of magnitude and so proper resolution of the solution - usually a certain fixed number of grid points per wave length implies quasi-optimality of the method.

When studying acoustic wave-propagation, it is often assumed to have constant material properties such as density and speed of sound, while in real complex materials, such as composites, these may be heterogeneous. Therefore, in this paper we study a multiscale Petrov-Galerkin method for the Helmholtz equation with large wave numbers $k$ and possibly heterogeneous material coefficients as a generalization of [6,13]. Standard first-order piecewise polynomials on the scale $H$ serve as trial functions in this method, whereas the test functions involve a correction by solutions to coercive fine-scale problems on the scale $h$. It turns out that these corrector functions, though having global support in the domain $\Omega$, exhibit an exponential decay away from the respective sources. Therefore, the corrector problems can be localized to a small domain whose width $m H$ is described by the oversampling parameter $m$. These local problems are translation invariant. Therefore, in periodic media only a small number of corrector problems must be solved depending on the number of local mesh configurations.

The stability of the method requires that the stability constant of the continuous operator depends polynomially on $k$. Such results are very rare in the literature even for the case of homogeneous media. We shall emphasize that such an assumption does not hold true in general [2]. The first positive estimates of this type go back to [11] for convex planar domains with pure Robin boundary. They were later generalized to other settings and three spatial dimensions in $[3,9]$. For instance, in the particular case of pure impedance boundary conditions with $\partial \Omega=\Gamma_{R}$, it was proved in $[3,5,11]$, by employing a technique of [10], that the inf-sup constant is bounded, i.e. $\gamma(k, \Omega) \lesssim k$. Further setups allowing for polynomially well-posedness in the presence of a single star-shaped sound-soft scatterer are described in [9]. For multiple scattering and, in particular, for scattering in heterogeneous media, the situation is completely open. To show that the assumption is satisfiable for non-trivial heterogeneous media, in this work we determine a class of smooth heterogeneous coefficients that allow for explicit-in- $k$ stability estimates. 


\subsection{Heterogeneous Helmholtz Problem}

We begin with some standard notation on complex-valued Lebesgue and Sobolev spaces that applies throughout this paper. The bar indicates complex conjugation and $i$ is the imaginary unit. The $L^{2}$ inner product is denoted by $(v, w)_{L^{2}(\Omega)}:=\int_{\Omega} v \bar{w} d x$. The Sobolev space of complex-valued $L^{p}$ functions over a domain $\omega$ whose generalized derivatives up to order $l$ belong to $L^{p}$ is denoted by $W^{l, p}(\omega)$ and $H^{l}(\omega):=W^{l, 2}(\omega)$. Further, the notation $A \lesssim B$ abbreviates $A \leq C B$ for some constant $C$ that is independent of the meshsize, the wave number $k$, and all further parameters in the method like the oversampling parameter $m$ or the fine-scale mesh-size $h ; A \approx B$ abbreviates $A \lesssim B \lesssim A$.

We now begin with some notation and problem setting. Let $\Omega \subset \mathbb{R}^{d}$ be an open bounded Lipschitz domain with polyhedral boundary for $d \geq 1$. We wish to find a solution $u$ that satisfies

$$
-\operatorname{div}(A(x) \nabla u)-k^{2} V^{2}(x) u=f \text { in } \Omega,
$$

along with the boundary conditions

$$
\begin{aligned}
u & =0 \text { on } \Gamma_{D}, \\
A(x) \nabla u \cdot \nu & =0 \text { on } \Gamma_{N}, \\
A(x) \nabla u \cdot \nu-i k \beta(x) u & =g \text { on } \Gamma_{R} .
\end{aligned}
$$

Here, $\nu$ denotes the outer normal to $\partial \Omega=\overline{\Gamma_{D} \cup \Gamma_{N} \cup \Gamma_{R}}$, where the boundary sections are assumed disjoint. We suppose that $\left|\Gamma_{R}\right|>0$, but allow the other portions of the boundary to have measure zero. Although the results in this paper hold for a weaker dual space here we suppose $f \in L^{2}(\Omega)$ and $g \in L^{2}\left(\Gamma_{R}\right)$. For the coefficients, we suppose $A(x), V^{2}(x) \in W^{1, \infty}(\Omega)$, and $\beta(x) \in L^{\infty}(\Omega)$ are real valued. Moreover, we suppose there exist positive constants $A_{\min }, A_{\max }, \beta_{\min }, \beta_{\max }, V_{\min }$, and $V_{\max }$ independent of $k$ such that for all $x \in \Omega$ we have

$$
\begin{aligned}
A_{\min } & \leq A(x) \leq A_{\max } \\
\beta_{\min } & \leq \beta(x) \leq \beta_{\max } \\
V_{\min }^{2} & \leq V^{2}(x) \leq V_{\max }^{2}
\end{aligned}
$$

We denote the space

$$
V:=\left\{u \in H^{1}(\Omega) \mid u=0 \text { on } \Gamma_{D}\right\}
$$

and denote the norm weighted with $A(x), V(x)$, and $k$ to be for $\omega \subset \Omega$. 


$$
\|u\|_{V, \omega}:=\sqrt{\|k V u\|_{L^{2}(\omega)}^{2}+\left\|A^{\frac{1}{2}} \nabla u\right\|_{L^{2}(\omega)}^{2}},
$$

where if $\omega=\Omega$, we simply write $\|u\|_{V}$. We have the following variational form corresponding to (1): Find $u \in V$ such that

$$
a(u, v)=(f, v)_{L^{2}(\Omega)}+(g, v)_{L^{2}\left(\Gamma_{R}\right)} \text { for all } v \in V
$$

where the complex-valued sesquilinear form $a: V \times V \rightarrow \mathbb{C}$ is given by

$$
a(u, v)=(A(x) \nabla u, \nabla v)_{L^{2}(\Omega)}-\left(k^{2} V^{2}(x) u, v\right)_{L^{2}(\Omega)}-(i k \beta(x) u, v)_{L^{2}\left(\Gamma_{R}\right)} .
$$

Here we write $(u, v)_{L^{2}(\Omega)}=\int_{\Omega} u \bar{v} d x$ and similarly $(u, v)_{L^{2}\left(\Gamma_{R}\right)}=\int_{\Gamma_{R}} u \bar{v} d s$.

\subsection{Motivation for a Multiscale Method}

It is well known [1] that the pollution effect cannot be avoided in standard methods. However, it may be overcome by coupling the polynomial degree of the method with the wave number $k$ [12]. Therefore, multiscale methods appear to be a natural tool to incorporate fine-scale features in a low-order discretizations. Moreover, the parameters of this method must be coupled logarithmically with the wave number and therefore requires the stability constant of the continuous problem to be polynomially dependent of $k$ to arrive at a computationally efficient method. Hence, the stability of the continuous heterogeneous problem (1) is critical to the analysis of the related algorithms. In general, it is often shown (or possibly assumed) that there exists some constant $C_{\text {stab }}(k, \Omega)>0$, which depends on $k$, the geometry, and the coefficients, such that

$$
\|u\|_{V} \leq C_{s t a b}(k, \Omega)\left(\|f\|_{L^{2}(\Omega)}+\|g\|_{L^{2}\left(\Gamma_{R}\right)}\right) .
$$

Further, turning to the inf-sup type lower bound. It is often shown, or possibly assumed, that there exists some constant $\gamma(k, \Omega)$, related to $C_{s t a b}(k, \Omega)$, such that

$$
\gamma(k, \Omega)^{-1} \leq \inf _{v \in V \backslash\{0\}} \sup _{w \in V \backslash\{0\}} \frac{\operatorname{Re} a(v, w)}{\|v\|_{V}\|w\|_{V}} .
$$

As noted, it is often the case that these constants depend merely polynomially on $k$. However, it has been demonstrated that there are special instances of exponential $k$ dependence on $C_{\text {stab }}(k, \Omega)$ [2], and thus, highly unstable inf-sup constants $\gamma(k, \Omega)$. 


\section{Stability of the Heterogeneous Helmholtz Model}

As discussed in Section 1, the stability and regularity of the continuous problem has been investigated for constant coefficients in various contexts. In this section, we shall investigate the stability of the continuous problem with respect to wave number in the case of heterogeneous coefficients. We proceed using the variational techniques with geometric constraints [9].

As noted in Section 1, in the case of constant coefficients, there exists various methods to bound $\gamma(k, \Omega)$ in terms of $k$. Most importantly, the possible exponential dependence discussion in [2], will be excluded here. We will show in Section 2, that for certain classes of coefficients, we are able to obtain a favorable polynomial bound for $\gamma(k, \Omega)$. To this end, we will employ variational techniques and so-called Rellich type identities with restrictions on the types of geometries similar to the work of [9] and references therein.

As we use the variational techniques we will make the geometric assumptions made by [9]. That is we suppose that there exists a $x_{0} \in \mathbb{R}^{d}$ and a $\eta>0$ such that

$$
\begin{aligned}
& \left(x-x_{0}\right) \cdot \nu \leq 0 \text { on } \Gamma_{D}, \\
& \left(x-x_{0}\right) \cdot \nu=0 \text { on } \Gamma_{N}, \\
& \left(x-x_{0}\right) \cdot \nu \geq \eta \text { on } \Gamma_{R} .
\end{aligned}
$$

For a summary of such possible domains, we refer the reader to [9]. However, to get some sense of a geometry the reader may envision a convex domain with pure impedance boundary conditions. This of course may be weakened.

\subsection{Statement of Stability, Connections to Inf-Sup Constants, and Boundedness}

In this section we present our main stability result. The variational techniques employed require assumptions on the class of coefficients to remain valid. We outline these constraints and obtain a bounded-in- $k$ result. We further relate these to the inf-sup constants and explore the boundedness of the non-constant coefficient case.

Theorem 1. Suppose $\Omega \subset \mathbb{R}^{d}$, is a bounded connected Lipschitz domain and satisfies the geometric assumptions (9). Let $u$ be a solution of (1) with the boundary conditions (2), coefficients satisfying the bounds (3), and $k \geq k_{0}>0$, for some $k_{0}$. Further, we suppose the regularity $u \in H^{3 / 2+\delta}(\Omega)$ for $\delta>0$.

Define the following function

$$
S(x):=\operatorname{div}\left(\left(\frac{V^{2}(x)}{A(x)}\right)\left(x-x_{0}\right)\right)
$$


and further, we will denote $C_{G}$ to be the minimal constant so that

$$
2\left|\int_{\Omega}\left(\frac{\nabla A}{A}\right) \nabla u\left(\left(x-x_{0}\right) \cdot \nabla \bar{u}\right) d x\right| \leq C_{G}\left\|\left(\frac{\nabla A}{A}\right)\right\|_{L^{\infty}(\Omega)}\|\nabla u\|_{L^{2}(\Omega)}^{2} .
$$

We suppose that

$$
\begin{aligned}
& S_{\text {min }}=\min _{x \in \Omega} S(x)>0, \\
& S_{\text {min }}-\left((d-2)+C_{G}\left\|\left(\frac{\nabla A}{A}\right)\right\|_{L^{\infty}(\Omega)}\right) \frac{V_{\text {max }}^{2}}{A_{\text {min }}}>0 .
\end{aligned}
$$

We then have the following estimate

$$
\|u\|_{V}^{2} \leq C^{*}\left(1+\frac{1}{k^{2}}\right)\left(\|f\|_{L^{2}(\Omega)}^{2}+\|g\|_{L^{2}\left(\Gamma_{R}\right)}^{2}\right)
$$

where $C^{*}$ depends only on the (3) and $\Omega$, but not on $k$.

Proof. See Appendix below.

Now that we have an explicit bound for a class of constant variable coefficients, we now will relate the constant $C_{s t a b}(k, \Omega):=C^{*}\left(1+\frac{1}{k^{2}}\right)$ to $\gamma(k, \Omega)$ given by (8).

Theorem 2. Supposing the assumptions in Theorem 1, we have the following estimate

$$
k^{-1} \lesssim\left(\gamma^{H}\right)^{-1} \lesssim \inf _{v \in V \backslash\{0\}} \sup _{w \in V \backslash\{0\}} \frac{\operatorname{Re} a(v, w)}{\|v\|_{V}\|w\|_{V}} .
$$

Where, $\gamma^{H}:=\left(1+C^{*}\left(k+\frac{1}{k}\right) V_{\max }^{2}\right)$.

Proof. We proceed by a standard argument from [5], adapted to the heterogeneous case. Given $u \in H^{1}(\Omega)$, define $z \in H^{1}(\Omega)$ as the solution of

$$
2 k^{2}\left(v, V^{2} u\right)_{L^{2}(\Omega)}=a(v, z), \text { for all } v \in V .
$$

Then, from the estimate (13), we have

$$
\|z\|_{V} \leq C^{*}\left(1+\frac{1}{k^{2}}\right) V_{\max }^{2} k^{2}\|u\|_{L^{2}(\Omega)} .
$$

Note that

$$
\operatorname{Re} a(u, u)=(A(x) \nabla u, \nabla u)_{L^{2}(\Omega)}-\left(k^{2} V^{2}(x) u, u\right)_{L^{2}(\Omega)} .
$$

and using (15), we and taking $v=u+z$ implies

$$
\operatorname{Re} a(u, v)=\operatorname{Re} a(u, u)+\operatorname{Re} a(u, z)=\|u\|_{V}^{2} .
$$


Using (16) we obtain

$$
\begin{aligned}
\|v\|_{V} \leq & \|u\|_{V}+\|z\|_{V} \leq\|u\|_{V}+C^{*}\left(1+\frac{1}{k^{2}}\right) V_{\max }^{2} k^{2}\|u\|_{L^{2}(\Omega)} \\
& \leq\left(1+C^{*}\left(k+\frac{1}{k}\right) V_{\max }^{2}\right)\|u\|_{V} .
\end{aligned}
$$

Hence, $\operatorname{Re} a(u, v)=\|u\|_{V}^{2} \geq\left(1+C^{*}\left(k+\frac{1}{k}\right) V_{\max }^{2}\right)^{-1}\|v\|_{V}\|u\|_{V}$, taking

$$
\gamma^{H}:=\left(1+C^{*}\left(k+\frac{1}{k}\right) V_{\max }^{2}\right) \approx k
$$

yields the result.

Finally, for completeness, we include a brief proof of the boundedness of the variational from.

Theorem 3. Supposing the assumptions in Theorem 1, the variational form (6) has the following boundedness property

$$
|a(u, v)| \leq C_{a}\|u\|_{V}\|v\|_{V},
$$

Here $C_{a}$ may depend on the bounds (3), multiplicative trace constants, and $\Omega$, but not $k$.

Proof. From the variational form we have

$$
\begin{aligned}
&|a(u, v)| \leq\left|\left(A^{\frac{1}{2}} \nabla u, A^{\frac{1}{2}} \nabla v\right)_{L^{2}(\Omega)}\right|+\left|(k V u, k V v)_{L^{2}(\Omega)}\right| \\
&+\left|\left((\beta k)^{\frac{1}{2}} u,(\beta k)^{\frac{1}{2}} v\right)_{L^{2}\left(\Gamma_{R}\right)}\right| \\
& \leq\left\|A^{\frac{1}{2}} \nabla u\right\|_{L^{2}(\Omega)}\left\|A^{\frac{1}{2}} \nabla v\right\|_{L^{2}(\Omega)}+\|k V u\|_{L^{2}(\Omega)}\|k V v\|_{L^{2}(\Omega)} \\
&+\left\|(\beta k)^{\frac{1}{2}} u\right\|_{L^{2}\left(\Gamma_{R}\right)}\left\|(\beta k)^{\frac{1}{2}} v\right\|_{L^{2}\left(\Gamma_{R}\right)} \\
& \lesssim\|u\|_{V}\|v\|_{V}+\left\|(\beta k)^{\frac{1}{2}} u\right\|_{L^{2}\left(\Gamma_{R}\right)}\left\|(\beta k)^{\frac{1}{2}} v\right\|_{L^{2}\left(\Gamma_{R}\right)}
\end{aligned}
$$

We have from the multiplicative trace inequality

$$
\begin{aligned}
\left\|k^{\frac{1}{2}} u\right\|_{L^{2}\left(\Gamma_{R}\right)}^{2} & \leq C_{M}\left(\left\|k^{\frac{1}{2}} u\right\|_{L^{2}(\Omega)}\left|k^{\frac{1}{2}} u\right|_{H^{1}(\Omega)}+\operatorname{diam}(\Omega)^{-1}\left\|k^{\frac{1}{2}} u\right\|_{L^{2}(\Omega)}^{2}\right) \\
& \leq C_{M}\left(\|k u\|_{L^{2}(\Omega)}^{2}+|u|_{H^{1}(\Omega)}^{2}+\operatorname{diam}(\Omega)^{-1}\left\|k^{\frac{1}{2}} u\right\|_{L^{2}(\Omega)}^{2}\right) \\
& \lesssim C_{M}\left(\|u\|_{V}+\operatorname{diam}(\Omega)^{-1}\|k u\|_{L^{2}(\Omega)}^{2}\right) \lesssim C_{M}\|u\|_{V}^{2}
\end{aligned}
$$

since $k \geq 1$. Applying this to the $\Gamma_{R}$ terms we arrive at (18). 


\section{The Multiscale Method}

In this section, we will introduce the the notation on finite element spaces and meshes that defines the multiscale Petrov-Galerkin method (msPGFEM) for the heterogeneous Helmholtz problem. As noted, this method is based on ideas in an algorithm developed for homogeneous coefficients in [13], and later presented in the Petrov-Galerkin framework [6]. We will stay in line with the notation and presentation of [6], as this is the basis for the algorithm applied to a heterogeneous media. We begin by defining the basic components needed, then define the multiscale method as well as some computational aspects. Finally, we will briefly discuss the error analysis for the method, however, this will not differ too far from the homogeneous coefficient algorithm and as thus, will refer the reader to technical proofs in [6].

\subsection{Meshes and Data Structures}

We begin with the basic notation needed regarding the relevant mesh and data structures. For the sake of clarity and completeness, we will briefly recall the notation used in [6]. Let $\mathcal{G}_{H}$ be a regular partition of $\Omega$ into intervals, parallelograms, parallelepipeds for $d=1,2,3$, respectively, such that $\bigcup \mathcal{G}_{H}=\bar{\Omega}$ and any two distinct $T, T^{\prime} \in \mathcal{G}_{H}$ are either disjoint or share exactly one lower-dimensional hyper-face (that is a vertex or an edge for $d \in\{2,3\}$ or a face for $d=3)$. We suppose the mesh is shape-regular, that is the aspect ratio of the elements in $\mathcal{G}_{H}$ is uniformly bounded. For simplicity, we are considering quadrilaterals (resp. hexahedra) with parallel faces, this guarantees the non-degeneracy of the elements in $\mathcal{G}_{H}$. Again, the theory of this paper carries over to partitions satisfying suitable non-degeneracy conditions or even to meshless methods based on proper partitions of unity [8].

Given any subdomain $S \subseteq \bar{\Omega}$, we define its neighborhood to be

$$
\mathrm{N}(S):=\operatorname{int}\left(\cup\left\{T \in \mathcal{G}_{H}: T \cap \bar{S} \neq \emptyset\right\}\right) .
$$

Furthermore, we introduce for any $m \geq 2$ the patch extensions

$$
\mathrm{N}^{1}(S):=\mathrm{N}(S) \quad \text { and } \quad \mathrm{N}^{m}(S):=\mathrm{N}\left(\mathrm{N}^{m-1}(S)\right) .
$$

Note that the shape-regularity implies that there is a uniform bound denoted $C_{\mathrm{ol}, m}$, on the number of elements in the $m$ th-order patch, $\#\left\{K \in \mathcal{G}_{H}: K \subseteq\right.$ $\left.\overline{\mathrm{N}^{m}(T)}\right\} \leq C_{\mathrm{ol}, m}$ for all $T \in \mathcal{G}_{H}$. We will abbreviate $C_{\mathrm{ol}}:=C_{\mathrm{ol}, 1}$. Further, we assume that the coarse-scale mesh $\mathcal{G}_{H}$ is quasi-uniform. This implies that $C_{\mathrm{ol}, m}$ depends polynomially on $m$. The global mesh-size is $H:=\max \{\operatorname{diam}(T)\}$ for all $T \in \mathcal{G}_{H}$. 
We will denote $Q_{p}\left(\mathcal{G}_{H}\right)$ to be the space of piecewise polynomials of partial degree less than or equal to $p$. The space of globally continuous piecewise first-order polynomials is given by $\mathcal{S}^{1}\left(\mathcal{G}_{H}\right):=C^{0}(\Omega) \cap Q_{1}\left(\mathcal{G}_{H}\right)$, and by incorporating the Dirichlet condition we arrive at the standard $Q_{1}$ finite element space denoted here as

$$
V_{H}:=\mathcal{S}^{1}\left(\mathcal{G}_{H}\right) \cap V .
$$

The set of free vertices, or the degrees of freedom, is denoted by

$$
\mathcal{N}_{H}:=\left\{z \in \bar{\Omega}: z \text { is a vertex of } \mathcal{G}_{H} \text { and } z \notin \Gamma_{D}\right\} .
$$

To construct our fine-scale and thus, multiscale spaces we will need to define a coarse-grid quasi-interpolation operator. For simplicity of presentation,we suppose here that this quasi-interpolation is also projective. This assumption may be lifted c.f. [8] and references therein. We let $I_{H}: V \rightarrow V_{H}$ be a surjective quasi-interpolation operator that acts as a stable quasi-local projection in the sense that $I_{H}^{2}=I_{H}$ and that for any $T \in \mathcal{G}_{H}$ and all $v \in V$ the following local stability result holds

$$
H^{-1}\left\|v-I_{H} v\right\|_{L^{2}(T)}+\left\|\nabla I_{H} v\right\|_{L^{2}(T)} \leq C_{I_{H}}\|\nabla v\|_{L^{2}(\mathrm{~N}(T))} .
$$

Under the mesh condition that

$$
k H \lesssim 1,
$$

is bounded by a generic constant, this implies stability in the $\|\cdot\|_{V}$ norm

$$
\left\|I_{H} v\right\|_{V} \leq C_{I_{H}, V}\|v\|_{V} \quad \text { for all } v \in V,
$$

with a $k$-independent constant $C_{I_{H}, V}$. However, $C_{I_{H}, V}$, will depend on the constants in (3).

One possible choice and which we use in our implementation of the method, is to define $I_{H}:=E_{H} \circ \Pi_{H}$, where $\Pi_{H}$ is the piecewise $L^{2}$ projection onto $Q_{1}\left(\mathcal{G}_{H}\right)$ and $E_{H}$ is the averaging operator that maps $Q_{1}\left(\mathcal{G}_{H}\right)$ to $V_{H}$ by assigning to each free vertex the arithmetic mean of the corresponding function values of the neighbouring cells, that is, for any $v \in Q_{1}\left(\mathcal{G}_{H}\right)$ and any free vertex $z \in \mathcal{N}_{H}$,

$$
\left(E_{H}(v)\right)(z)=\left.\sum_{\substack{T \in \mathcal{G}_{H} \\ \text { with } z \in T}} v\right|_{T}(z) / \#\left\{K \in \mathcal{G}_{H}: z \in K\right\} .
$$

Note that with this choice of quasi-interpolation, $\left.E_{H}(v)\right|_{\Gamma_{D}}=0$ by construction. For this choice, the proof of (19) follows from combining the well-established approximation and stability properties of $\Pi_{H}$ and $E_{H}$ shown in [4]. 


\subsection{Definition of the Method}

The multiscale method is determined by three parameters, namely the coarsescale mesh-size $H$, the fine-scale mesh-size $h$, and the oversampling parameter $m$. We assign to any $T \in \mathcal{G}_{H}$ its $m$-th order patch $\Omega_{T}:=\mathrm{N}^{m}(T), m \in \mathbb{N}$, and define for any $v, w \in V$ the localized sesquilinear forms of (6) to $\Omega_{T}$ as

$a_{\Omega_{T}}(u, v)=(A(x) \nabla u, \nabla v)_{L^{2}\left(\Omega_{T}\right)}-\left(k^{2} V^{2}(x) u, v\right)_{L^{2}\left(\Omega_{T}\right)}-(i k \beta(x) u, v)_{L^{2}\left(\Gamma_{R} \cap \partial \Omega_{T}\right)}$.

and to $T$, we have

$a_{\Omega_{T}}(u, v)=(A(x) \nabla u, \nabla v)_{L^{2}(T)}-\left(k^{2} V^{2}(x) u, v\right)_{L^{2}(T)}-(i k \beta(x) u, v)_{L^{2}\left(\Gamma_{R} \cap \partial T\right)}$.

Let the fine-scale mesh $\mathcal{G}_{h}$, be a global uniform refinement of the mesh $\mathcal{G}_{H}$ over $\Omega$ and define

$$
V_{h}\left(\Omega_{T}\right):=\left\{v \in Q_{1}\left(\mathcal{G}_{h}\right) \cap V: v=0 \text { outside } \Omega_{T}\right\} .
$$

Define the null space

$$
W_{h}\left(\Omega_{T}\right):=\left\{v_{h} \in V_{h}\left(\Omega_{T}\right): I_{H}\left(v_{h}\right)=0\right\}
$$

of the quasi-interpolation operator $I_{H}$ defined in the previous section. This is the space often referred to as the fine-scale or small-scale space. Given any nodal basis function $\Lambda_{z} \in V_{H}$, let $\lambda_{z, T} \in W_{h}\left(\Omega_{T}\right)$ solve the subscale corrector problem

$$
a_{\Omega_{T}}\left(w, \lambda_{z, T}\right)=a_{T}\left(w, \Lambda_{z}\right) \text { for all } w \in W_{h}\left(\Omega_{T}\right) .
$$

Let $\lambda_{z}:=\sum_{T \in \mathcal{G}_{H}} \lambda_{z, T}$ and define the multiscale test function

$$
\widetilde{\Lambda}_{z}:=\Lambda_{z}-\lambda_{z}
$$

The space of multiscale test functions then reads

$$
\widetilde{V}_{H}:=\operatorname{span}\left\{\widetilde{\Lambda}_{z}: z \in \mathcal{N}_{H}\right\}
$$

We emphasize that the dimension of the multiscale space is the same as the original coarse space, $\operatorname{dim} V_{H}=\operatorname{dim} \widetilde{V}_{H}$. Moreover, it is independent of the parameters $m$ and $h$. Finally, the multiscale Petrov-Galerkin FEM seeks to find $u_{H} \in V_{H}$ such that

$$
a\left(u_{H}, \tilde{v}_{H}\right)=\left(f, \tilde{v}_{H}\right)_{L^{2}(\Omega)}+\left(g, \tilde{v}_{H}\right)_{L^{2}\left(\Gamma_{R}\right)} \quad \text { for all } \tilde{v}_{H} \in \tilde{V}_{H}
$$

As in [6], the error analysis and the numerical experiments will show that the choice $H \lesssim k^{-1}, m \approx \log (k)$ will be sufficient to guarantee stability and quasi-optimality properties, provided that $k^{\alpha} h \lesssim 1$ where $\alpha$ depends on the stability and regularity of the continuous problem. This constant $\alpha$ was the 
subject of Section 2. The conditions on $h$ are the same as for the standard $Q_{1}$ FEM on the global fine scale. For example, in 2 dimensions, in the case of pure Robin boundary conditions on a convex domain, it is required that $k^{3 / 2} h \lesssim 1$ for stability [14] and $k^{2} h \lesssim 1$ for quasi-optimality [11] is required.

\section{Error Analysis}

The error analysis for the algorithm presented in Section 3, is very similar to that developed in [13] and references therein, and in particular for the Petrov-Galerkin formulation we discuss now in [6]. It is clear the proofs are unaffected by the coefficients as the arguments rely on very general constants being bounded such as $C_{a}, C_{\text {stab }}(k, \Omega)$, and $\gamma(k, \Omega)$, for example. This is primarily due to the upper and lower boundedness on the coefficients (3). However, we will highlight the main themes of the analysis here as this will be useful to refer to in our discussion on Numerical Examples in Section 5 as well as general completeness of the discussion.

We begin the error analysis with some notation. We denote the global finite element space on the fine scale by $V_{h}:=V_{h}(\Omega)=\mathcal{S}^{1}\left(\mathcal{G}_{h}\right) \cap V$. We denote the solution operator of the truncated element corrector problem (21) by $\mathcal{C}_{T, m}$. Then, any $z \in \mathcal{N}_{H}$ and any $T \in \mathcal{G}_{H}$ satisfy $\lambda_{z, T}=\mathcal{C}_{T, m}\left(\Lambda_{z}\right)$ and we refer to $\mathcal{C}_{T, m}$ as the truncated element correction operator. The map $\Lambda_{z} \mapsto \lambda_{z}$ described in Subsection 3.2 defines a linear operator $\mathcal{C}_{m}$ via $\mathcal{C}_{m}\left(\Lambda_{z}\right)=\lambda_{z}$ for any $z \in \mathcal{N}_{H}$, referred to as correction operator.

For the analysis we introduce idealized counterparts of these correction operators where the patch $\Omega_{T}$ equals $\Omega$. These global corrections are never computed and are merely used in the analysis. We define the null space $W_{h}:=\left\{v \in V_{h}: I_{H}(v)=0\right\}$, also referred to as the fine-scale space on the global domain. For any $v \in V$, the idealized element corrector problem seeks $\mathcal{C}_{T, \infty} v \in W_{h}$ such that

$$
a\left(w, \mathcal{C}_{T, \infty} v\right)=a_{T}(w, v) \quad \text { for all } w \in W_{h} .
$$

Furthermore, define

$$
\mathcal{C}_{\infty} v:=\sum_{T \in \mathcal{G}_{H}} \mathcal{C}_{T, \infty} v
$$

Recall, we proved in Section 2 that the form $a$ with heterogeneous coefficients given by (6), is continuous and there is a constant $C_{a}$ such that

$$
a(v, w) \leq C_{a}\|v\|_{V}\|w\|_{V} \quad \text { for all } v, w \in V .
$$

The following result implies the well-posedness of the idealized corrector problems.

\section{Lemma 1 (Well-posedness for idealized corrector problems).}


Provided

$$
C_{I_{H}} \sqrt{C_{\mathrm{ol}}} H k \leq 1
$$

we have for all $w \in W_{h}$ equivalence of norms

$$
A_{\text {min }}^{\frac{1}{2}}\|\nabla w\|_{L^{2}(\Omega)} \leq\|w\|_{V} \leq\left(V_{\max }^{2}+A_{\max }\right)^{\frac{1}{2}}\|\nabla w\|_{L^{2}(\Omega)},
$$

and coercivity

$$
\left(V_{\max }^{2}+A_{\max }\right)\|\nabla w\|_{L^{2}(\Omega)}^{2} \leq \operatorname{Re} a(w, w) .
$$

Proof. The lower bound is trivial, indeed we have that

$$
\|w\|_{V}^{2}=\|k V w\|_{L^{2}(\Omega)}^{2}+\left\|A^{\frac{1}{2}} \nabla w\right\|_{L^{2}(\Omega)}^{2} \geq A_{\text {min }}\|\nabla w\|_{L^{2}(\Omega)}^{2} .
$$

For the upper bound, we note for any $w \in W_{h}$ the property (19) implies

$$
k^{2}\|V w\|_{L^{2}(\Omega)}^{2}=k^{2}\left\|V\left(1-I_{H}\right) w\right\|_{L^{2}(\Omega)}^{2} \leq V_{\max }^{2} C_{I_{H}}^{2} C_{\mathrm{ol}} H^{2} k^{2}\|\nabla w\|_{L^{2}(\Omega)}^{2} .
$$

Thus, using (25) we arrive at

$$
\begin{aligned}
\|w\|_{V}^{2} & =\|k V w\|_{L^{2}(\Omega)}^{2}+\left\|A^{\frac{1}{2}} \nabla w\right\|_{L^{2}(\Omega)}^{2} \\
& \leq V_{\max }^{2} C_{I_{H}}^{2} C_{\mathrm{ol}} H^{2} k^{2}\|\nabla w\|_{L^{2}(\Omega)}^{2}+A_{\max }\|\nabla w\|_{L^{2}(\Omega)}^{2} \\
& \leq\left(V_{\max }^{2}+A_{\max }\right)\|\nabla w\|_{L^{2}(\Omega)}^{2} .
\end{aligned}
$$

Note from this we have

$$
\begin{aligned}
\|k V w\|_{L^{2}(\Omega)}^{2} & \leq\left(V_{\max }^{2}+A_{\max }\right)\|\nabla w\|_{L^{2}(\Omega)}^{2}-\left\|A^{\frac{1}{2}} \nabla w\right\|_{L^{2}(\Omega)}^{2} \\
& \leq\left(V_{\max }^{2}+A_{\max }-A_{\min }\right)\|\nabla w\|_{L^{2}(\Omega)}^{2},
\end{aligned}
$$

and so

$$
\begin{aligned}
\operatorname{Re} a(w, w) & =\left\|A^{\frac{1}{2}} \nabla w\right\|_{L^{2}(\Omega)}^{2}-\|k V w\|_{L^{2}(\Omega)}^{2} \\
& \geq\left(V_{\text {max }}^{2}+A_{\max }\right)\|\nabla w\|_{L^{2}(\Omega)}^{2} .
\end{aligned}
$$

Thus, equivalence and coercivity is proven.

Lemma 1 implies that the idealized corrector problems (24) are well-posed and the correction operator $\mathcal{C}_{\infty}$ is continuous in the sense that

$$
\left\|\mathcal{C}_{\infty} v_{H}\right\|_{V} \leq C_{\mathcal{C}}\left\|v_{H}\right\|_{V} \quad \text { for all } v_{H} \in V_{H}
$$

for some constant $C_{\mathcal{C}} \approx 1$. Since the inclusion $W_{h}\left(\Omega_{T}\right) \subseteq W_{h}$ holds, the well-posedness result of Lemma 1 carries over to the corrector problems (21) in the subspace $W_{h}\left(\Omega_{T}\right)$ with the sesquilinear form $a_{\Omega_{T}}$.

Again as with the homogeneous coefficient case [6], the proof of wellposedness of the Petrov-Galerkin method (22) is based on the fact that the 
difference $\left(\mathcal{C}_{\infty}-\mathcal{C}_{m}\right)(v)$ decays exponentially with the distance from $\operatorname{supp}(v)$. In the next theorem, we quantify the difference between the idealized and the discrete correctors. As the proof is a bit technical and does not differ fundamentally from the homogeneous case, we refer the reader to Appendix of [6] and references therein. The proof is based on the exponential decay of the corrector $\mathcal{C}_{\infty} \Lambda_{z}$ and requires the resolution condition (25), namely $k H \lesssim 1$.

Theorem 4. Under the resolution condition (25), there exist constants $C_{1} \approx$ $1 \approx C_{2}$ and $0<\theta<1$ such that any $v \in V_{H}$, any $T \in \mathcal{G}_{H}$ and any $m \in \mathbb{N}$ satisfy

$$
\begin{aligned}
\left\|\nabla\left(\mathcal{C}_{T, \infty} v-\mathcal{C}_{T, m} v\right)\right\|_{L^{2}(\Omega)} & \leq C_{1} \theta^{m}\|\nabla v\|_{L^{2}(T)} \\
\left\|\nabla\left(\mathcal{C}_{\infty} v-\mathcal{C}_{m} v\right)\right\|_{L^{2}(\Omega)} & \leq C_{2} \sqrt{C_{\mathrm{ol}, m}} \theta^{m}\|\nabla v\|_{L^{2}(\Omega)} .
\end{aligned}
$$

Proof. See Appendix of [6].

Provided we choose the fine-mesh $h$ is chosen small enough, the standard finite element over the mesh $\mathcal{G}_{h}$ is stable in the sense that there exists a constant $C_{\mathrm{FEM}}$ such that with $\gamma(k, \Omega)$ from (8) it holds that

$$
\left(C_{\mathrm{FEM}} \gamma(k, \Omega)\right)^{-1} \leq \inf _{v \in V_{h} \backslash\{0\}} \sup _{w \in V_{h} \backslash\{0\}} \frac{\operatorname{Re} a(v, w)}{\|v\|_{V}\|w\|_{V}} .
$$

Recall, this is actually a condition on the fine-scale parameter $h$. In general, the requirements on $h$ depend on the stability of the continuous problem [11]. We now recall the conditions on the oversampling parameter for the wellposedness of the discrete problem. Again, the proof here does not rely heavily on the coefficients, just the general boundedness and ellipticity constants etc. Thus, we again refer the reader to [6].

Theorem 5 (Well-posedness of the discrete problem). Under the resolution conditions (25) and (28) and the following oversampling condition

$$
m \gtrsim\left|\log \left(C_{\mathrm{FEM}} \gamma(k, \Omega)\right)\right| /|\log (\theta)|,
$$

problem (22) is well-posed and the constant $C_{\mathrm{PG}}:=2 C_{I_{H}, V} C_{\mathcal{C}} C_{\mathrm{FEM}}$ satisfies

$$
\left(C_{\mathrm{PG}} \gamma(k, \Omega)\right)^{-1} \leq \inf _{v_{H} \in V_{H} \backslash\{0\}} \sup _{\tilde{v}_{H} \in \widetilde{V}_{H} \backslash\{0\}} \frac{\operatorname{Re} a\left(v_{H}, \tilde{v}_{H}\right)}{\left\|v_{H}\right\|_{V}\left\|\tilde{v}_{H}\right\|_{V}} .
$$

Proof. See [6].

The quasi-optimality requires the following additional condition on the oversampling parameter $m$,

$$
m \gtrsim\left|\log \left(C_{\mathrm{PG}} \gamma(k, \Omega)\right)\right| /|\log (\theta)| .
$$


Theorem 6 (Quasi-optimality). The resolution conditions (25) and (28) and the oversampling conditions (29) and (30) imply that the solution $u_{H}$ to (22) with parameters $H, h$, and $m$ and the solution $u_{h}$ of the standard Galerkin FEM on the mesh $\mathcal{G}_{h}$ satisfy

$$
\left\|u_{h}-u_{H}\right\|_{V} \lesssim\left\|\left(1-I_{H}\right) u_{h}\right\|_{V} \approx \min _{v_{H} \in V_{H}}\left\|u_{h}-v_{H}\right\|_{V}
$$

Proof. See [6].

The following consequence of Theorem 6 states an estimate for the error $u-u_{H}$.

Corollary 1. Under the conditions of Theorem 6, the discrete solution $u_{H}$ to $(22)$ satisfies with some constant $C \approx 1$ that

$$
\left\|u-u_{H}\right\|_{V} \leq\left\|u-u_{h}\right\|_{V}+C \min _{v_{H} \in V_{H}}\left\|u_{h}-v_{H}\right\|_{V}
$$

\section{Numerical Examples}

In this section, we will provide a few examples that satisfy the assumptions on the coefficients (12). Hence, the set of bounded smooth coefficients that yields polynomial-in- $k$ bounds is non-trivial. We show that for some coefficients, as the oscillations become more frequent we violate the conditions (12). In particular, it appears that the restriction on the amplitude of the coefficients is related to the restrictions on the frequency of oscillations. We then proceed to implement the multiscale mathod on a smooth coefficient for both cases when the conditions are satisfied and when it is violated. Further, we implement the method on discontinuous periodic coefficients to highlight broader applicability of the method.

\subsection{Example Coefficients}

To simplify things, yet provide non-trivial coefficients, we will only consider radially symmetric conditions in $\mathbb{R}^{2}$. Indeed, even with this symmetry, we are able to highlight the complexities and restrictiveness in these conditions. We will see that the frequency of oscillations play a considerable role in violation of these conditions, as well as the amplitude.

We take $\Omega \subset \mathbb{R}^{2}$ to be given by the unit circle $\Omega:=\left\{(x, y) \in \mathbb{R}^{2} \mid x^{2}+y^{2} \leq\right.$ $1\}$ and $\partial \Omega=\left\{(x, y) \in \mathbb{R}^{2} \mid x^{2}+y^{2}=1\right\}$. Further, we will take $\Gamma_{N}=\Gamma_{D}=\emptyset$, so that $\Gamma_{R}=\partial \Omega$. We take $x_{0}=(0,0) \in \Omega$, and so $m=\left(x-x_{0}\right)=r \hat{r}$, where $r^{2}=x^{2}+y^{2}$ and $\hat{r}$ is the standard unit normal in radial coordinates. Then, clearly, $m \cdot \nu=1$ on $\Gamma_{R}$ and so the geometric assumptions (9) are 
satisfied with this domain. We will take $\beta(x)=1, g(x)=0$, and suppose that $f:=f(r)$, is a given radially symmetric forcing. We finally suppose that the heterogeneities are radially symmetric, $V^{2}(x)=V^{2}(r)$, and $A(x)=A(r)$. We briefly recall in radial coordinates that for $A=\left(A_{r}, A_{\theta}\right)$

$$
\begin{aligned}
\operatorname{div}(A) & =\frac{1}{r} \frac{\partial}{\partial r}\left(r A_{r}\right)+\frac{1}{r} \frac{\partial A_{\theta}}{\partial \theta} . \\
\nabla A & =\frac{\partial A_{r}}{\partial r} \hat{r}+\frac{1}{r} \frac{\partial A_{\theta}}{\partial \theta} \hat{\theta} . \\
\int_{\Omega} A d x d y & =\int_{0}^{2 \pi} \int_{0}^{1} A r d r d \theta,
\end{aligned}
$$

where $\hat{\theta}$ is the standard angular coordinate. By examining the conditions (12), we are able to produce a few interesting examples.

Case 1: $A=1$. Note that from condition (12b), that if $A=1$ (or constant), we see that the conditions simplify slightly since the gradient terms in $A$ will vanish. Indeed, now we see that only condition (12a) must be satisfied. In this setting, we must have that $\operatorname{div}\left(V^{2} m\right)>0$ for our estimates to hold, or rewritten in radial coordinates as

$$
\frac{1}{r} \frac{\partial}{\partial r}\left(V^{2}(r) r^{2}\right)>0
$$

From this condition we may choose a few possible coefficients for $V(r)$. A trivial example is when $V^{2}(r)=r+1$. Clearly,

$$
\frac{1}{r} \frac{\partial}{\partial r}\left(r^{3}+r^{2}\right)=\frac{1}{r}\left(3 r^{2}+2 r\right)=3 r+2>0 .
$$

Many such polynomial in $r$ choices exist as long as they do not violate boundedness and positivity.

More interesting examples come from oscillatory coefficients. Suppose, for $\epsilon>0$, we take now the innocent looking example

$$
V^{2}(r)=\frac{1}{2} \sin \left(\frac{2 \pi r}{\epsilon}\right)+5
$$

and so

$$
\frac{1}{r} \frac{\partial}{\partial r}\left(\frac{r^{2}}{2} \sin \left(\frac{2 \pi r}{\epsilon}\right)+5 r^{2}\right)=\sin \left(\frac{2 \pi r}{\epsilon}\right)+\frac{r \pi}{\epsilon} \cos \left(\frac{2 \pi r}{\epsilon}\right)+10 .
$$

A quick investigation shows that if $\epsilon=1$, then (31) is satisfied, however, when $\epsilon=.1$ it is violated. Hence, if the coefficient becomes highly oscillatory, the stability condition is not satisfied. Also note that if we fix $\epsilon=1$, but extend the domain from a unit circle to one of radius $R$, we will eventually enter a 
negative region. Hence, the domain size also may have an effect on stability from the viewpoint of conditions (12).

Case 2: $A=V^{2}$. Turning to the definition of $S(x)$ in (10), we see that if $A=V^{2}$, the functions simplifies to $S(x)=d$. Thus, condition (12a) is always satisfied. For $d=2$, (12b) becomes

$$
2-\left(C_{G}\left\|\left(\frac{\nabla A}{A}\right)\right\|_{L^{\infty}(\Omega)}\right) \frac{A_{\max }}{A_{\min }}>0 .
$$

Taking a closer look at the terms related to $C_{G}$ from the Appendix below, we have in radial coordinates

$$
\begin{aligned}
2\left|\int_{\Omega}\left(\frac{\nabla A}{A}\right) \nabla u\left(\left(x-x_{0}\right) \cdot \nabla \bar{u}\right) d x\right| & =2\left|\int_{0}^{2 \pi} \int_{0}^{1}\left(\frac{r^{2}}{A(r)} \frac{\partial A(r)}{\partial r}\right)\left(\frac{\partial u(r)}{\partial r}\right)^{2} d r d \theta\right| \\
& \leq 2\left\|\frac{1}{A(r)} \frac{\partial A(r)}{\partial r}\right\|_{L^{\infty}(\Omega)}\|\nabla u\|_{L^{2}(\Omega)}^{2} .
\end{aligned}
$$

Hence, we may take here $C_{G}=2$. Noting that

$$
\frac{\partial}{\partial r} \ln (A)=\frac{1}{A(r)} \frac{\partial A(r)}{\partial r},
$$

then the condition (34) becomes

$$
1-\left(\left\|\frac{\partial}{\partial r} \ln (A)\right\|\right) \frac{A_{\max }}{A_{\min }}>0 .
$$

Taking

$$
V^{2}(r)=A(r)=\exp \left(\alpha\left(\sin \left(\frac{r}{\epsilon}\right)+\delta\right)\right),
$$

for $\epsilon, \alpha$, and $\delta$ positive, then

$$
\left\|\frac{\partial}{\partial r} \ln (A)\right\|_{L^{\infty}(\Omega)}=\left\|\frac{\alpha}{\epsilon} \cos \left(\frac{r}{\epsilon}\right)\right\|_{L^{\infty}(\Omega)}=\frac{\alpha}{\epsilon} .
$$

Note further that $A_{\max }=\exp (\alpha(\delta+1))$ and $A_{\min }=\exp (\alpha(\delta-1))$, and so $\frac{A_{\max }}{A_{\min }}=\exp (2 \alpha)$. Hence,

$$
1-\left(\left\|\frac{\partial}{\partial r} \ln (A)\right\|\right) \frac{A_{\max }}{A_{\min }}=1-\frac{\alpha}{\epsilon} \exp (2 \alpha)>0 .
$$

or $\alpha \exp (2 \alpha)<\epsilon$. We see from this calculation that the frequency of oscillation in the coefficients is related to the amplitude as far as the conditions (12) are 
concerned. The more oscillatory the function, the smaller the amplitude must be in this example.

\subsection{Results}

We now present the results from out numerical experiments. We give 3 example coefficients; based on (32), (36), and a discontinuous example. In all three experiments we took $\Omega=(-1,1)^{2}$ to be the unit square. We use triangular meshes and continuous $P_{1}$ finite elements as trial functions. We used $k=2^{5}$, $g=0$, and the approximate point source

$$
f(x)= \begin{cases}\exp \left(-\frac{1}{1-(20|x|)^{2}}\right) & \text { for }|x|<1 / 20 \\ 0 & \text { else. }\end{cases}
$$

The coarse-scale mesh-sizes are $H=2^{-3}, 2^{-4}, 2^{-5}, 2^{-6}$ and the fine-scale mesh-size is $h=2^{-8}$.

The convergence history plots display the errors in the $\|\cdot\|_{V}$ norm as well as $L^{2}$ norms. We compare the multiscale Petrov-Galerkin method for oversampling parameters $m=1,2,3$ with the standard $P_{1}$ finite element method and the best-approximation. To compute the error quantity we take the standard finite element solution at the fine scale $h$ to be the overkill solution.

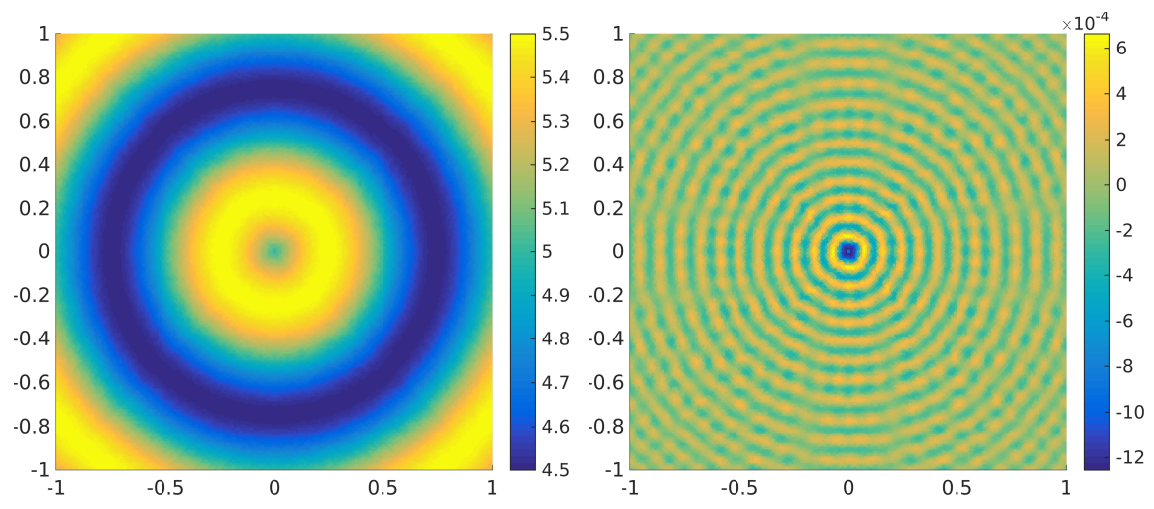

Fig. 1 The coefficient $V^{2}$ for example 1. Fig. 2 Plot of the solution for example 1.

For the first example, we take $A=1$ and $V^{2}$ as (32). with $\epsilon=1$ and refer to this as example 1 . Note that this does not violate the stability condition. The coefficient $V^{2}$ is displayed in Figure 1 and the corresponding computational solution is displayed in Figure 2. Figures 3-4 display the convergence history in the $V$-norm and the $L^{2}$ norm for example 1 . In general, we see that for the 
method appears to perform much better than the corresponding standard $P_{1}$ finite element. However, there appears to be some resonance effects of some sort that is particularly pronounced in the $V$ norm.
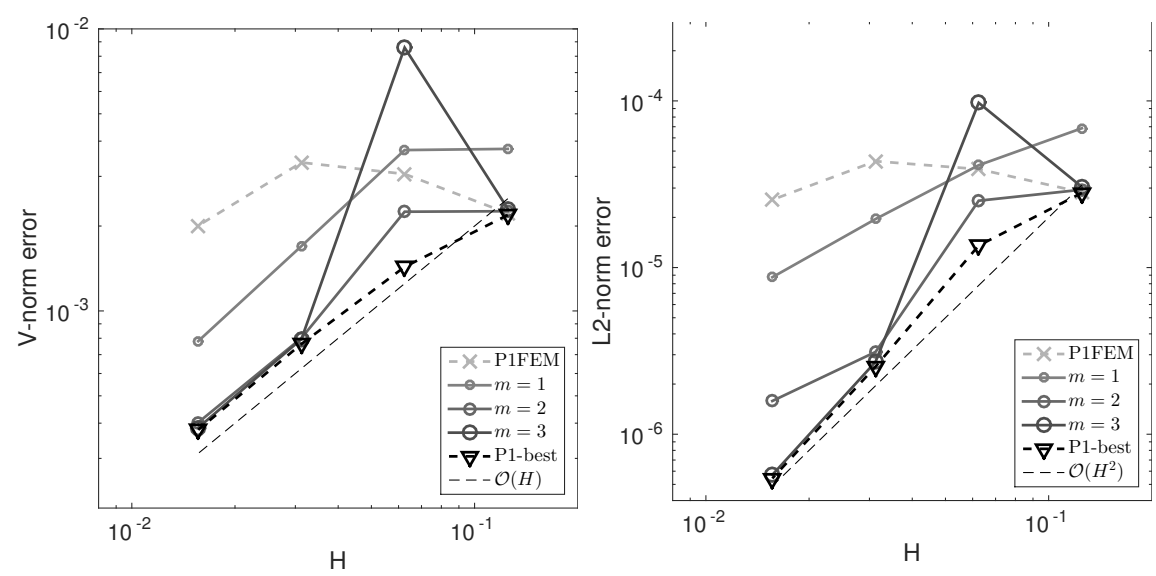

Fig. 3 Convergence in $V$ norm: example 1. Fig. 4 Convergence in $L^{2}$ norm: example 1.

For the second example, we take $A=V^{2}$ and $V^{2}$ as (36), and refer to this as example 2. For the parameters we took $\delta=1, \epsilon=0.1, \alpha=0.08$, and note that the corresponding stability condition $\alpha \exp (2 \alpha)<\epsilon$ is narrowly satisfied. The coefficient $V^{2}$ is displayed in Figure 5 and the computational solution

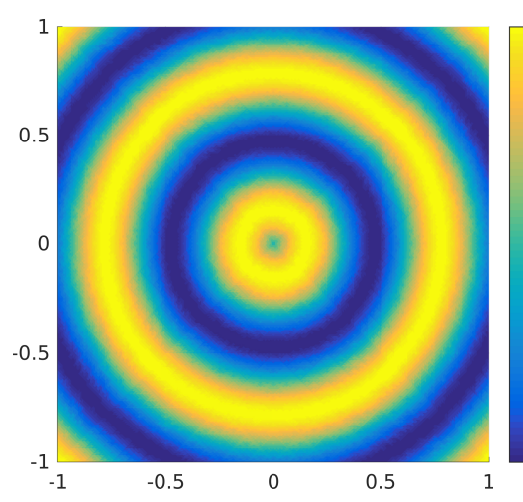

Fig. 5 The coefficient $V^{2}$ for example 2.

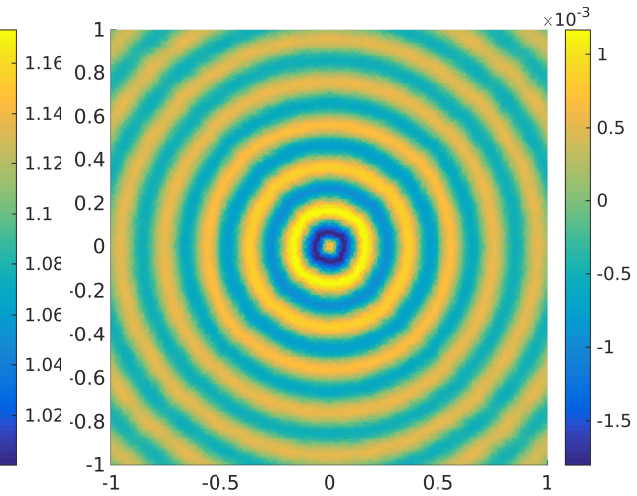

Fig. 6 Plot of the solution for example 2.

is displayed in Figure 6. Figures 7-8 display the convergence history in the $V$-norm and the $L^{2}$ norm for example 2 . We see that in this example, we 
achieve faster convergence and do not see the resonance effects. This is also the case for the standard finite elements.

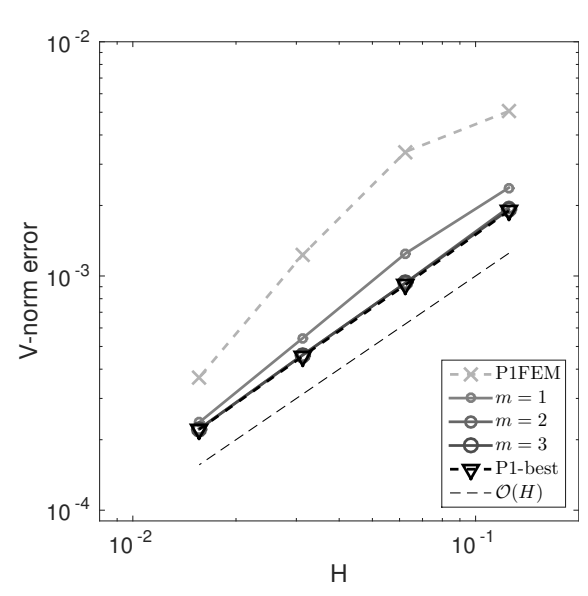

Fig. 7 Convergence in $V$ norm example 2 .

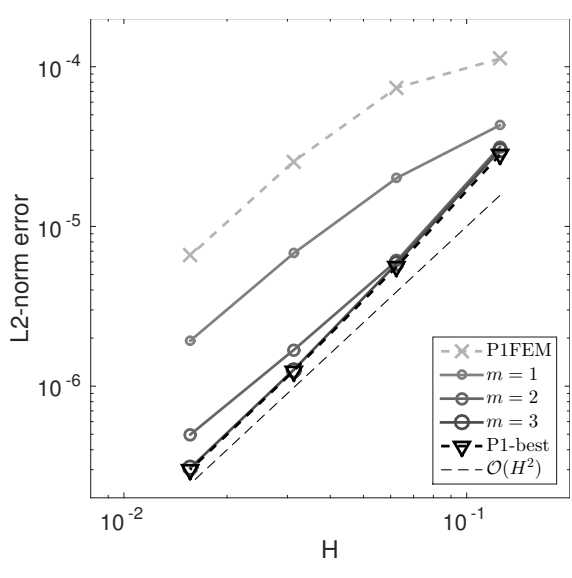

Fig. 8 Convergence in $L^{2}$ norm example 2 .

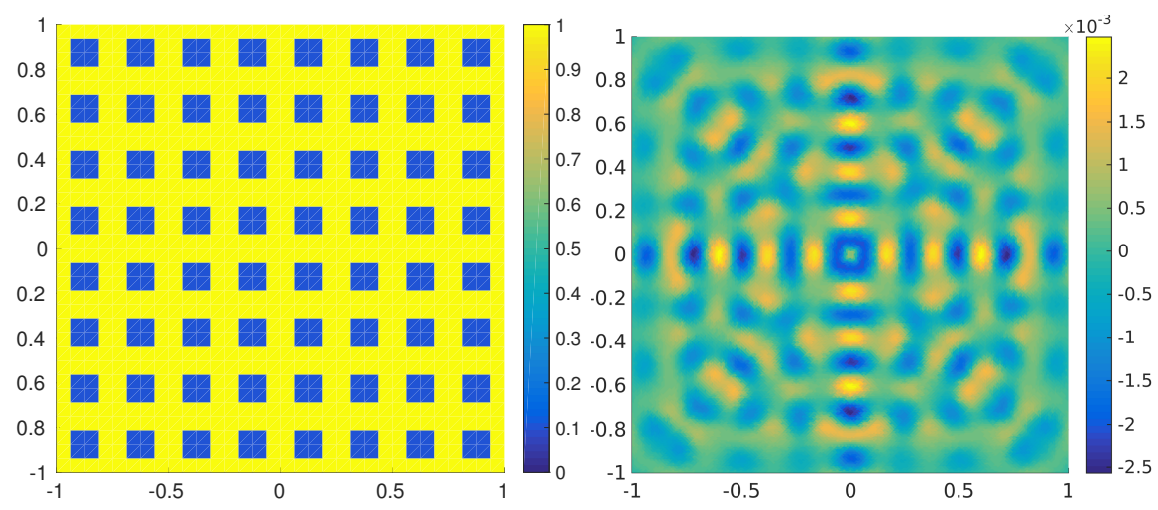

Fig. 9 The coefficient $V^{2}$ for example 3. Fig. 10 Plot of the solution for example 3.

We now present a numerical example outside of our stability theory. We take $V^{2}=1$ except at periodically placed blocks where $V^{2}=0$ and plot the function in Figure 9. We refer to this as example 3. The computational solution is displayed in Figure 10. Figures 11-12 display the convergence history in the $V$-norm and the $L^{2}$ norm for example 3 . We observe that the method performs particularly well in this example, especially when compared against the corresponding $P_{1}$ finite element. We do not see the resonances as with example 1. 


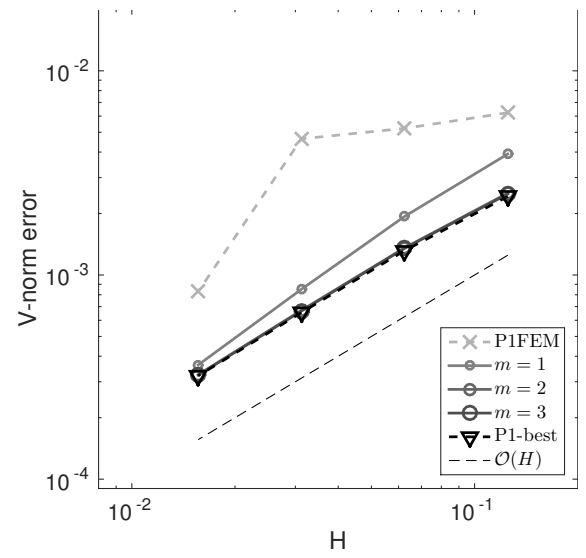

Fig. 11 Convergence in $V$ norm example 3. Fig. 12 Convergence in $L^{2}$ norm example

\section{Conclusions}

In this work, we developed a multiscale method to efficiently solve the heterogeneous Helmholtz equation at high frequency. The primary challenge was establishing $k$-explicit bounds for the continuous problem as these are critical in the analysis of the patch truncation parameter. We established these bounds for a class of smooth coefficients given some restrictions that appear to depend heavily on the frequency of oscillations and the amplitude of the coefficients. We then presented our multiscale method whose error analysis is not significantly modified by the heterogeneities assuming standard upper and lower boundedness. Finally, we implemented the algorithm on two coefficients that fit inside the class of coefficients in our main theorem and one that is discontinuous. We see that the method performs well in these cases. Future work includes exploring if these stability estimates apply to a greater class of more heterogeneous coefficients with less smoothness.

\section{Appendix: Proof of Stability}

\section{Technical and Auxiliary Lemmas}

We will now proceed by recalling and demonstrating a few technical and auxiliary Lemmas used in the proof of Theorem 1. We begin with two critical technical lemmas that remain unchanged from the homogeneous case examined in [9] and are repeated here for completeness.

Lemma 2. Let $m \in W^{1, \infty}(\Omega)^{d}$ and for all $q \in H^{1}(\Omega)$ we have 


$$
\int_{\partial \Omega}|q|^{2} m \cdot \nu d s=\int_{\Omega} \operatorname{div}(m)|q|^{2} d x+2 \operatorname{Re} \int_{\Omega} q m \cdot \nabla \bar{q} d x .
$$

Proof. See [9], Lemma 3.1.

Lemma 3. Let $m \in W^{1, \infty}(\Omega)^{d}$ and for all $q \in H_{\Gamma_{D}}^{1}(\Omega) \cap H^{3 / 2+\delta}, \delta>0$, we have

$$
\begin{aligned}
& \int_{\partial \Omega \backslash \Gamma_{D}}|\nabla q|^{2} m \cdot \nu d s-\int_{\Gamma_{D}}\left|\partial_{\nu} q\right|^{2} m \cdot \nu d s \\
& =\int_{\Omega} \operatorname{div}(m)|\nabla q|^{2} d x-2 \operatorname{Re} \int_{\Omega} \nabla q \cdot(\nabla \bar{q} \nabla) m d x \\
& -2 \operatorname{Re} \int_{\Omega} \Delta q(m \cdot \nabla \bar{q}) d x+2 \operatorname{Re} \int_{\partial \Omega \backslash \Gamma_{D}} \partial_{\nu} q(m \cdot \nabla \bar{q}) d s
\end{aligned}
$$

Proof. See [7].

Here we will present a few auxiliary Lemmas.

Lemma 4. Let $\Omega \subset \mathbb{R}^{d}$ be a bounded connected Lipschitz domain. Let $u \in$ $H^{1}(\Omega)$ be a weak solution of (1), with $f \in L^{2}(\Omega)$ and $g \in L^{2}\left(\Gamma_{R}\right)$. Then, we have for any $\epsilon>0$

$$
k^{2}\|u\|_{L^{2}\left(\Gamma_{R}\right)}^{2} \leq \frac{1}{\beta_{\min }}\left(\frac{1}{\epsilon}\|f\|_{L^{2}(\Omega)}^{2}+k^{2} \epsilon\|u\|_{L^{2}(\Omega)}^{2}+\frac{1}{\beta_{\min }}\|g\|_{L^{2}\left(\Gamma_{R}\right)}^{2}\right) .
$$

Proof. Taking $v=u$ into the variational form (5) and looking at the imaginary part we have

$$
\Im(a(u, u))=-(k \beta(x) u, u)=\Im\left((g, u)_{L^{2}\left(\Gamma_{R}\right)}+(f, u)_{L^{2}(\Omega)}\right),
$$

and so

$$
\begin{aligned}
k \beta_{\min }\|u\|_{L^{2}\left(\Gamma_{R}\right)}^{2} & \leq\|u\|_{L^{2}(\Omega)}\|f\|_{L^{2}(\Omega)}+\|u\|_{L^{2}\left(\Gamma_{R}\right)}\|g\|_{L^{2}\left(\Gamma_{R}\right)} . \\
& \leq \frac{1}{2 k \xi_{1}}\|f\|_{L^{2}(\Omega)}^{2}+\frac{k \xi_{1}}{2}\|u\|_{L^{2}(\Omega)}^{2}+\frac{1}{2 \xi_{2}}\|g\|_{L^{2}\left(\Gamma_{R}\right)}^{2}+\frac{\xi_{2}}{2}\|u\|_{L^{2}\left(\Gamma_{R}\right)}^{2} .
\end{aligned}
$$

Multiplying by $k$, dividing by $\beta_{\text {min }}$, and setting $\xi_{2}=\beta_{\min } k$ we obtain

$$
\begin{aligned}
& k^{2}\|u\|_{L^{2}\left(\Gamma_{R}\right)}^{2} \\
& \leq \frac{1}{\beta_{\min }}\left(\frac{1}{2 \xi_{1}}\|f\|_{L^{2}(\Omega)}^{2}+\frac{k^{2} \xi_{1}}{2}\|u\|_{L^{2}(\Omega)}^{2}+\frac{1}{2 \beta_{\min }}\|g\|_{L^{2}\left(\Gamma_{R}\right)}^{2}+\frac{k^{2} \beta_{\min }}{2}\|u\|_{L^{2}\left(\Gamma_{R}\right)}^{2}\right),
\end{aligned}
$$

and we obtain

$$
\frac{k^{2}}{2}\|u\|_{L^{2}\left(\Gamma_{R}\right)}^{2} \leq \frac{1}{\beta_{\min }}\left(\frac{1}{2 \xi_{1}}\|f\|_{L^{2}(\Omega)}^{2}+\frac{k^{2} \xi_{1}}{2}\|u\|_{L^{2}(\Omega)}^{2}+\frac{1}{2 \beta_{\min }}\|g\|_{L^{2}\left(\Gamma_{R}\right)}^{2}\right) .
$$


Taking $\xi_{1}=\epsilon>0$ we arrive at the estimate.

We will also need the estimate below.

Lemma 5. Let $\Omega \subset \mathbb{R}^{d}$ be a bounded connected Lipschitz domain. Let $u \in$ $H^{1}(\Omega)$ be a weak solution of (1) with $f \in L^{2}(\Omega)$ and $g \in L^{2}\left(\Gamma_{R}\right)$. Then, we have

$$
\begin{aligned}
& \|\nabla u\|_{L^{2}(\Omega)}^{2} \\
& \leq \frac{1}{A_{\min }}\left[k^{2}\left(V_{\max }^{2}+\frac{\xi_{4}}{\beta_{\min }}+\frac{\xi_{3}}{2}\right)\|u\|_{L^{2}(\Omega)}^{2}\right. \\
& \left.\quad+\left(\frac{1}{2 k^{2} \xi_{3}}+\frac{1}{\beta_{\min } \xi_{4}}\right)\|f\|_{L^{2}(\Omega)}^{2}+\left(\frac{1}{\beta_{\min }^{2}}+\frac{1}{4 k^{2}}\right)\|g\|_{L^{2}\left(\Gamma_{R}\right)}^{2}\right] .
\end{aligned}
$$

for any $\xi_{3}, \xi_{4}>0$.

Proof. Taking $v=u$ into the variational form (5) and looking at the real part we have

$$
\begin{aligned}
\operatorname{Re}(a(u, u)) & =(A(x) \nabla u, \nabla u)_{L^{2}(\Omega)}-\left(k^{2} V^{2}(x) u, u\right)_{L^{2}(\Omega)} \\
& =\operatorname{Re}\left((g, u)_{L^{2}\left(\Gamma_{R}\right)}+(f, u)_{L^{2}(\Omega)}\right)
\end{aligned}
$$

and so we have

$$
\left\|A^{\frac{1}{2}} \nabla u\right\|_{L^{2}(\Omega)}^{2} \leq k^{2}\|V u\|_{L^{2}(\Omega)}^{2}+\|u\|_{L^{2}(\Omega)}\|f\|_{L^{2}(\Omega)}+\|u\|_{L^{2}\left(\Gamma_{R}\right)}\|g\|_{L^{2}\left(\Gamma_{R}\right)} .
$$

Using the maximal and minimal values we have for any $\xi_{3}>0$ that

$$
\begin{aligned}
A_{\min }\|\nabla u\|_{L^{2}(\Omega)}^{2} & \leq k^{2}\|V u\|_{L^{2}(\Omega)}^{2}+\|u\|_{L^{2}(\Omega)}\|f\|_{L^{2}(\Omega)}+\|u\|_{L^{2}\left(\Gamma_{R}\right)}\|g\|_{L^{2}\left(\Gamma_{R}\right)} \\
& \leq\left(k^{2} V_{\max }^{2}+\frac{k^{2} \xi_{3}}{2}\right)\|u\|_{L^{2}(\Omega)}^{2}+\frac{1}{2 k^{2} \xi_{3}}\|f\|_{L^{2}(\Omega)}^{2} \\
& +\frac{1}{4 k^{2}}\|g\|_{L^{2}\left(\Gamma_{R}\right)}^{2}+k^{2}\|u\|_{L^{2}\left(\Gamma_{R}\right)}^{2} .
\end{aligned}
$$

Using estimate (40) we may write for any $\epsilon>0$

$$
k^{2}\|u\|_{L^{2}\left(\Gamma_{R}\right)}^{2} \leq \frac{1}{\beta_{\min }}\left(k^{2} \epsilon\|u\|_{L^{2}(\Omega)}^{2}+\frac{1}{\epsilon}\|f\|_{L^{2}(\Omega)}^{2}+\frac{1}{\beta_{\min }}\|g\|_{L^{2}\left(\Gamma_{R}\right)}^{2}\right) .
$$

Inserting the above inequality into (42) we obtain 


$$
\begin{aligned}
A_{\min } \| & \nabla u \|_{L^{2}(\Omega)}^{2} \\
& \leq\left(k^{2} V_{\max }^{2}+\frac{k^{2} \xi_{3}}{2}\right)\|u\|_{L^{2}(\Omega)}^{2}+\frac{1}{2 k^{2} \xi_{3}}\|f\|_{L^{2}(\Omega)}^{2}+\frac{1}{4 k^{2}}\|g\|_{L^{2}\left(\Gamma_{R}\right)}^{2} \\
& +\frac{1}{\beta_{\min }}\left(k^{2} \epsilon\|u\|_{L^{2}(\Omega)}^{2}+\frac{1}{\epsilon}\|f\|_{L^{2}(\Omega)}^{2}+\frac{1}{\beta_{\min }}\|g\|_{L^{2}\left(\Gamma_{R}\right)}^{2}\right) .
\end{aligned}
$$

Taking $\epsilon=\xi_{4}$ the above inequality becomes

$$
\begin{aligned}
A_{\min }\|\nabla u\|_{L^{2}(\Omega)}^{2} & \leq k^{2}\left(V_{\text {max }}^{2}+\frac{\xi_{4}}{\beta_{\min }}+\frac{\xi_{3}}{2}\right)\|u\|_{L^{2}(\Omega)}^{2} \\
& +\left(\frac{1}{2 k^{2} \xi_{3}}+\frac{1}{\beta_{\min \xi_{4}}}\right)\|f\|_{L^{2}(\Omega)}^{2}+\left(\frac{1}{\beta_{\text {min }}^{2}}+\frac{1}{4 k^{2}}\right)\|g\|_{L^{2}\left(\Gamma_{R}\right)}^{2} .
\end{aligned}
$$

Thus, we obtained our estimate.

\section{Proof of Main Stability Result}

We are now in a position to prove Theorem 1. The key observation is that the Laplacian may be rewritten using (1) and combined with the technical and auxiliary lemmas. This leads to the conditions on the coefficients (12).

Proof (Proof of Theorem 1).

Using (39) where we write

$$
-\Delta u=\frac{1}{A}\left(f+k^{2} V^{2} u+\nabla A \cdot \nabla u\right),
$$

$\partial_{\nu} u=0$ on $\Gamma_{N}$, and $\partial_{\nu} u=i k \beta u+g$ on $\Gamma_{R}$, we obtain

$$
\begin{aligned}
& \int_{\partial \Omega \backslash \Gamma_{D}}|\nabla u|^{2} m \cdot \nu d s-\int_{\Gamma_{D}}\left|\partial_{\nu} u\right|^{2} m \cdot \nu d s \\
& =\int_{\Omega} \operatorname{div}(m)|\nabla u|^{2} d x-2 \operatorname{Re} \int_{\Omega} \nabla u \cdot(\nabla \bar{u} \nabla) m d x \\
& \quad+2 \operatorname{Re} \int_{\Omega} \frac{1}{A}\left(f+k^{2} V^{2} u+\nabla A \cdot \nabla u\right)(m \cdot \nabla \bar{u}) d x \\
& \quad+2 \operatorname{Re} \int_{\Gamma_{R}}(i k \beta u+g)(m \cdot \nabla \bar{u}) d s .
\end{aligned}
$$

Using (38) with the transform $m \rightarrow \frac{V^{2}}{A} m$, we have 


$$
\begin{aligned}
k^{2} \int_{\partial \Omega}|u|^{2} & \left(\frac{V^{2}}{A}\right) m \cdot \nu d s \\
& =k^{2} \int_{\Omega} \operatorname{div}\left(\frac{V^{2}}{A} m\right)|u|^{2} d x+2 k^{2} \operatorname{Re} \int_{\Omega} u\left(\frac{V^{2}}{A}\right) m \cdot \nabla \bar{u} d x .
\end{aligned}
$$

Using this to replace the term $\operatorname{Re} \int_{\Omega}\left(\frac{V^{2}}{A}\right) u(m \cdot \nabla \bar{u}) d x$, we have

$$
\begin{aligned}
& \int_{\partial \Omega \backslash \Gamma_{D}}|\nabla u|^{2} m \cdot \nu d s-\int_{\Gamma_{D}}\left|\partial_{\nu} u\right|^{2} m \cdot \nu d s \\
& =\int_{\Omega} \operatorname{div}(m)|\nabla u|^{2} d x-2 \operatorname{Re} \int_{\Omega} \nabla u \cdot(\nabla \bar{u} \nabla) m d x \\
& \quad+2 \operatorname{Re} \int_{\Omega}\left(\frac{f}{A}\right)(m \cdot \nabla \bar{u}) d x+2 \operatorname{Re} \int_{\Omega}\left(\frac{\nabla A}{A}\right) \cdot \nabla u(m \cdot \nabla \bar{u}) d x \\
& \quad+2 \operatorname{Re} \int_{\Gamma_{R}}(i k \beta u+g)(m \cdot \nabla \bar{u}) d s \\
& \quad-k^{2} \int_{\Omega} \operatorname{div}\left(\frac{V^{2}}{A} m\right)|u|^{2} d x+k^{2} \int_{\partial \Omega}|u|^{2}\left(\frac{V^{2}}{A}\right) m \cdot \nu d s .
\end{aligned}
$$

Expanding out the boundary terms in each of the portions we have

$$
\begin{aligned}
& -\int_{\Gamma_{D}}\left|\partial_{\nu} u\right|^{2} m \cdot \nu d s+\int_{\Gamma_{N}}|\nabla u|^{2} m \cdot \nu d s \\
& \quad+\int_{\Gamma_{R}}|\nabla u|^{2} m \cdot \nu d s+k^{2} \int_{\Omega} \operatorname{div}\left(\frac{V^{2}}{A} m\right)|u|^{2} d x \\
& =\int_{\Omega} \operatorname{div}(m)|\nabla u|^{2} d x-2 \operatorname{Re} \int_{\Omega} \nabla u \cdot(\nabla \bar{u} \nabla) m d x \\
& \quad+2 \operatorname{Re} \int_{\Omega}\left(\frac{f}{A}\right)(m \cdot \nabla \bar{u}) d x+2 \operatorname{Re} \int_{\Omega}\left(\frac{\nabla A}{A}\right) \cdot \nabla u(m \cdot \nabla \bar{u}) d x \\
& \quad+k^{2} \int_{\Gamma_{N}}|u|^{2}\left(\frac{V^{2}}{A}\right) m \cdot \nu d s+k^{2} \int_{\Gamma_{R}}|u|^{2}\left(\frac{V^{2}}{A}\right) m \cdot \nu d s \\
& \quad+2 \operatorname{Re} \int_{\Gamma_{R}}(i k \beta u+g)(m \cdot \nabla \bar{u}) d s .
\end{aligned}
$$

Now we suppose we make the geometric assumptions made by [9] outlined in (9). Recall, we have for $m=x-x_{0}$, thus we compute

$$
\begin{aligned}
\operatorname{div}\left(x-x_{0}\right) & =d \text { in } \Omega, \\
\nabla u \cdot(\nabla \bar{u} \nabla)\left(x-x_{0}\right) & =|\nabla u|^{2} \text { in } \Omega, \\
\left(x-x_{0}\right) \cdot \nu & \leq 0 \text { on } \Gamma_{D}, \\
\left(x-x_{0}\right) \cdot \nu & =0 \text { on } \Gamma_{N}, \\
\left(x-x_{0}\right) \cdot \nu & \geq \eta \text { on } \Gamma_{R} .
\end{aligned}
$$


Using the above relations in (45) we obtain

$$
\begin{aligned}
& \eta \int_{\Gamma_{R}}|\nabla u|^{2} d s+k^{2} \int_{\Omega} \operatorname{div}\left(\frac{V^{2}}{A}\left(x-x_{0}\right)\right)|u|^{2} d x \\
& \leq(d-2) \int_{\Omega}|\nabla u|^{2} d x+2 \operatorname{Re} \int_{\Omega}\left(\frac{f}{A}\right)\left(\left(x-x_{0}\right) \cdot \nabla \bar{u}\right) d x \\
& +2 \operatorname{Re} \int_{\Omega}\left(\frac{\nabla A}{A}\right) \nabla u\left(\left(x-x_{0}\right) \cdot \nabla \bar{u}\right) d x \\
& +k^{2} \int_{\Gamma_{R}}|u|^{2}\left(\frac{V^{2}}{A}\right)\left(x-x_{0}\right) \cdot \nu d s+2 \operatorname{Re} \int_{\Gamma_{R}}(i k \beta u+g)(m \cdot \nabla \bar{u}) d s .
\end{aligned}
$$

Recall, (10), where we define the following function

$$
\begin{aligned}
S(x) & :=\operatorname{div}\left(\left(\frac{V^{2}(x)}{A(x)}\right)\left(x-x_{0}\right)\right) \\
& =d\left(\frac{V^{2}(x)}{A(x)}\right)+\left(2 \frac{V(x) \nabla V(x)}{A(x)}-\frac{V^{2}(x) \nabla A(x)}{A^{2}(x)}\right) \cdot\left(x-x_{0}\right),
\end{aligned}
$$

and from (12), we have a minimum for $S(x)$ exists and is positive

$$
S_{\min }=\min _{x \in \Omega} S(x)>0 .
$$

Further, from (12), we have $C_{G}$ to be the minimal constant so that

$$
2\left|\int_{\Omega}\left(\frac{\nabla A}{A}\right) \nabla u\left(\left(x-x_{0}\right) \cdot \nabla \bar{u}\right) d x\right| \leq C_{G}\left\|\left(\frac{\nabla A}{A}\right)\right\|_{L^{\infty}(\Omega)}\|\nabla u\|_{L^{2}(\Omega)}^{2} .
$$

Returning to inequality (46), we obtain

$$
\begin{aligned}
& \eta\|\nabla u\|_{L^{2}\left(\Gamma_{R}\right)}^{2}+k^{2} S_{\min }\|u\|_{L^{2}(\Omega)}^{2} \\
& \leq(d-2)\|\nabla u\|_{L^{2}(\Omega)}^{2}+C_{G}\left\|\left(\frac{\nabla A}{A}\right)\right\|_{L^{\infty}(\Omega)}\|\nabla u\|_{L^{2}(\Omega)}^{2} \\
& \quad+C_{1}\left(\frac{1}{A_{\min }}\|f\|_{L^{2}(\Omega)}\|\nabla u\|_{L^{2}(\Omega)}+\|g\|_{L^{2}\left(\Gamma_{R}\right)}\|\nabla u\|_{L^{2}\left(\Gamma_{R}\right)}\right) \\
& \quad+C_{1}\left(k^{2}\left(\frac{V_{\max }^{2}}{A_{\min }}\right)\|u\|_{L^{2}\left(\Gamma_{R}\right)}^{2}+k\|\beta\|_{L^{\infty}\left(\Gamma_{R}\right)}\|u\|_{L^{2}\left(\Gamma_{R}\right)}\|\nabla u\|_{L^{2}\left(\Gamma_{R}\right)}\right),
\end{aligned}
$$

where $C_{1}$ is independent of $k$ and the bounds (3). Note that on the right hand side we have for any $\xi_{5}, \xi_{6}>0$ the terms 


$$
\begin{aligned}
k\|\beta\|_{L^{\infty}\left(\Gamma_{R}\right)}\|u\|_{L^{2}\left(\Gamma_{R}\right)}\|\nabla u\|_{L^{2}\left(\Gamma_{R}\right)} & \leq \frac{k^{2}}{2 \xi_{5}}\|u\|_{L^{2}\left(\Gamma_{R}\right)}^{2}+\frac{\xi_{5}}{2}\|\beta\|_{L^{\infty}\left(\Gamma_{R}\right)}^{2}\|\nabla u\|_{L^{2}\left(\Gamma_{R}\right)}^{2} \\
\|g\|_{L^{2}\left(\Gamma_{R}\right)}\|\nabla u\|_{L^{2}\left(\Gamma_{R}\right)} & \leq \frac{1}{2 \xi_{6}}\|g\|_{L^{2}\left(\Gamma_{R}\right)}^{2}+\frac{\xi_{6}}{2}\|\nabla u\|_{L^{2}\left(\Gamma_{R}\right)}^{2} .
\end{aligned}
$$

We choose $\xi_{5}, \xi_{6}$ so that

$$
\frac{\eta}{2}=C_{1} \frac{\xi_{5}}{2}\|\beta\|_{L^{\infty}\left(\Gamma_{R}\right)}^{2}=C_{1} \frac{\xi_{6}}{2}
$$

and so

$$
\frac{k^{2}}{2 \xi_{5}} \leq \frac{C_{1}}{2 \eta}\|\beta\|_{L^{\infty}\left(\Gamma_{R}\right)}^{2} k^{2}
$$

We then obtain

$$
\begin{aligned}
k^{2} S_{\min }\|u\|_{L^{2}(\Omega)}^{2} & \leq C_{1}\left(\left(\frac{C_{1}}{2 \eta}\|\beta\|_{L^{\infty}\left(\Gamma_{R}\right)}^{2}+\frac{V_{\max }^{2}}{A_{\min }}\right) k^{2}\|u\|_{L^{2}\left(\Gamma_{R}\right)}^{2}\right) \\
& +C_{1}\left(\frac{1}{A_{\min }}\|f\|_{L^{2}(\Omega)}\|\nabla u\|_{L^{2}(\Omega)}+\frac{C_{1}}{2 \eta}\|g\|_{L^{2}\left(\Gamma_{R}\right)}^{2}\right) \\
& +(d-2)\|\nabla u\|_{L^{2}(\Omega)}^{2}+C_{G}\left\|\left(\frac{\nabla A}{A}\right)\right\|_{L^{\infty}(\Omega)}\|\nabla u\|_{L^{2}(\Omega)}^{2} .
\end{aligned}
$$

Taking $C_{2}^{b d}=C_{1}\left(\frac{C_{1}}{2 \eta}\|\beta\|_{L^{\infty}\left(\Gamma_{R}\right)}^{2}+\frac{V_{m a x}^{2}}{A_{\min }}\right)$ and letting $\epsilon=\beta_{\min } \xi_{7} / C_{2}^{b d}$ in the inequality (40) we have the relation

$$
C_{2}^{b d} k^{2}\|u\|_{L^{2}\left(\Gamma_{R}\right)}^{2} \leq \frac{\left(C_{2}^{b d}\right)^{2}}{\beta_{\min }^{2} \xi_{7}}\|f\|_{L^{2}(\Omega)}^{2}+k^{2} \xi_{7}\|u\|_{L^{2}(\Omega)}^{2}+\frac{C_{2}^{b d}}{\beta_{\min }^{2}}\|g\|_{L^{2}\left(\Gamma_{R}\right)}^{2} .
$$

Applying this above inequality to (50), we obtain

$$
\begin{aligned}
k^{2}\left(S_{\text {min }}-\right. & \left.\xi_{7}\right)\|u\|_{L^{2}(\Omega)}^{2} \\
\leq C_{1} & \left(\frac{1}{A_{\min }}\|f\|_{L^{2}(\Omega)}\|\nabla u\|_{L^{2}(\Omega)}+\frac{C_{1}}{2 \eta}\|g\|_{L^{2}\left(\Gamma_{R}\right)}^{2}\right) \\
& +\left((d-2)+C_{G}\left\|\left(\frac{\nabla A}{A}\right)\right\|_{L^{\infty}(\Omega)}\right)\|\nabla u\|_{L^{2}(\Omega)}^{2} \\
& +\frac{\left(C_{2}^{b d}\right)^{2}}{\beta_{\text {min }}^{2} \xi_{7}}\|f\|_{L^{2}(\Omega)}^{2}+\frac{C_{2}^{b d}}{\beta_{\text {min }}^{2}}\|g\|_{L^{2}\left(\Gamma_{R}\right)}^{2} .
\end{aligned}
$$

Recall the estimate (41), with $C_{3}^{b d}=\left((d-2)+C_{G}\left\|\left(\frac{\nabla A}{A}\right)\right\|_{L^{\infty}(\Omega)}\right)$, and taking $\xi_{4}=\frac{\xi_{3}}{2}=\xi_{8}$ 


$$
\begin{aligned}
& C_{3}^{b d}\|\nabla u\|_{L^{2}(\Omega)}^{2} \\
& \leq \frac{C_{3}^{b d} k^{2}}{A_{\min }}\left(V_{\max }^{2}+\frac{\xi_{8}}{\beta_{\min }}+\xi_{8}\right)\|u\|_{L^{2}(\Omega)}^{2} \\
&+\frac{C_{3}^{b d}}{A_{\min }}\left(\frac{1}{4 k^{2} \xi_{8}}+\frac{1}{\beta_{\min } \xi_{8}}\right)\|f\|_{L^{2}(\Omega)}^{2}+\frac{C_{3}^{b d}}{A_{\min }}\left(\frac{1}{\beta_{\min }^{2}}+\frac{1}{4 k^{2}}\right)\|g\|_{L^{2}\left(\Gamma_{R}\right)}^{2} .
\end{aligned}
$$

and so, using the above estimate (52)we obtain

$$
\begin{aligned}
& k^{2}\left(S_{\min }-\xi_{7}-\frac{C_{3}^{b d}}{A_{\min }}\left(V_{\text {max }}^{2}+\frac{\xi_{8}}{\beta_{\min }}+\xi_{8}\right)\right)\|u\|_{L^{2}(\Omega)}^{2} \\
& \leq C_{1}\left(\frac{1}{A_{\min }}\|f\|_{L^{2}(\Omega)}\|\nabla u\|_{L^{2}(\Omega)}+\frac{C_{1}}{2 \eta}\|g\|_{L^{2}\left(\Gamma_{R}\right)}^{2}\right) \\
& +\frac{C_{3}^{b d}}{A_{\min }}\left(\frac{1}{4 k^{2} \xi_{8}}+\frac{1}{\beta_{\min } \xi_{8}}\right)\|f\|_{L^{2}(\Omega)}^{2}+\frac{C_{3}^{b d}}{A_{\min }}\left(\frac{1}{\beta_{\min }^{2}}+\frac{1}{4 k^{2}}\right)\|g\|_{L^{2}\left(\Gamma_{R}\right)}^{2} \\
& +\frac{\left(C_{2}^{b d}\right)^{2}}{\beta_{\min }^{2} \xi_{7}}\|f\|_{L^{2}(\Omega)}^{2}+\frac{C_{2}^{b d}}{\beta_{\min }^{2}}\|g\|_{L^{2}\left(\Gamma_{R}\right)}^{2} .
\end{aligned}
$$

Finally to deal with the remaining term on the right hand side that contains $\nabla u$, we note using (41), letting $\frac{\xi_{4}}{\beta_{\min }}=\frac{\xi_{3}}{2}=\frac{V_{\max }^{2}}{2}$, and multiplying by $\xi_{9} /\left(2 A_{\min }\right), \xi_{9}>0$, we obtain

$$
\begin{gathered}
\frac{\xi_{9}}{2 A_{\min }}\|\nabla u\|_{L^{2}(\Omega)}^{2} \\
\leq \frac{\xi_{9}}{2 A_{\min }^{2}}\left[2 V_{\text {max }}^{2} k^{2}\|u\|_{L^{2}(\Omega)}^{2}+\left(\frac{2}{\beta_{\text {min }}^{2} V_{\max }^{2}}+\frac{1}{2 k^{2} V_{\max }^{2}}\right)\|f\|_{L^{2}(\Omega)}^{2}\right. \\
\left.\quad+\left(\frac{1}{\beta_{\text {min }}^{2}}+\frac{1}{4 k^{2}}\right)\|g\|_{L^{2}\left(\Gamma_{R}\right)}^{2}\right],
\end{gathered}
$$

and so

$$
\begin{aligned}
\frac{1}{A_{\min }}\|f\|_{L^{2}(\Omega)}\|\nabla u\|_{L^{2}(\Omega)} \\
\leq \frac{1}{2 \xi_{9} A_{\min }}\|f\|_{L^{2}(\Omega)}^{2}+\frac{\xi_{9}}{2 A_{\min }}\|\nabla u\|_{L^{2}(\Omega)}^{2} \\
\leq \frac{\xi_{9} V_{\max }^{2}}{A_{\min }^{2}} k^{2}\|u\|_{L^{2}(\Omega)}^{2} \\
\quad+\left(\frac{1}{2 A_{\min } \xi_{9}}+\frac{\xi_{9}}{2 A_{\min }^{2}}\left(\frac{2}{\beta_{\min }^{2} V_{\max }^{2}}+\frac{1}{2 k^{2} V_{\max }^{2}}\right)\right)\|f\|_{L^{2}(\Omega)}^{2} \\
\quad+\frac{\xi_{9}}{2 A_{\min }^{2}}\left(\frac{1}{\beta_{\min }^{2}}+\frac{1}{4 k^{2}}\right)\|g\|_{L^{2}\left(\Gamma_{R}\right)}^{2} .
\end{aligned}
$$

Applying this into (53), we obtain 


$$
\begin{aligned}
& k^{2}\left(S_{\min }-\xi_{7}-\frac{C_{3}^{b d}}{A_{\min }}\left(V_{\max }^{2}+\frac{\xi_{8}}{\beta_{\min }}+\xi_{8}\right)-\frac{C_{1} \xi_{9} V_{\max }^{2}}{A_{\min }^{2}}\right)\|u\|_{L^{2}(\Omega)}^{2} \\
& \leq C_{1}\left(\frac{1}{2 A_{\min } \xi_{9}}+\frac{\xi_{9}}{2 A_{\min }^{2}}\left(\frac{2}{\beta_{\min }^{2} V_{\max }^{2}}+\frac{1}{2 k^{2} V_{\max }^{2}}\right)\right)\|f\|_{L^{2}(\Omega)}^{2} \\
& +C_{1}\left(\frac{C_{1}}{2 \eta}+\frac{\xi_{9}}{2 A_{\min }^{2}}\left(\frac{1}{\beta_{\min }^{2}}+\frac{1}{4 k^{2}}\right)\right)\|g\|_{L^{2}\left(\Gamma_{R}\right)}^{2} \\
& +\frac{C_{3}^{b d}}{A_{\min }}\left(\frac{1}{4 k^{2} \xi_{8}}+\frac{1}{\beta_{\min } \xi_{8}}\right)\|f\|_{L^{2}(\Omega)}^{2} \\
& +\frac{C_{3}^{b d}}{A_{\min }}\left(\frac{1}{\beta_{\min }^{2}}+\frac{1}{4 k^{2}}\right)\|g\|_{L^{2}\left(\Gamma_{R}\right)}^{2}+\frac{\left(C_{2}^{b d}\right)^{2}}{\beta_{\min }^{2} \xi_{7}}\|f\|_{L^{2}(\Omega)}^{2}+\frac{C_{2}^{b d}}{\beta_{\min }^{2}}\|g\|_{L^{2}\left(\Gamma_{R}\right)}^{2} .
\end{aligned}
$$

Hence, we see that the critical term is $S_{\min }-\frac{C_{3}^{b d} V_{\max }^{2}}{A_{\min }}$. Recall,

$$
C_{3}^{b d}:=\left((d-2)+C_{G}\left\|\left(\frac{\nabla A}{A}\right)\right\|_{L^{\infty}(\Omega)}\right),
$$

thus, from (12), we have

$$
S_{\text {min }}-\left((d-2)+C_{G}\left\|\left(\frac{\nabla A}{A}\right)\right\|_{L^{\infty}(\Omega)}\right) \frac{V_{\max }^{2}}{A_{\min }}>0 .
$$

Since (55) is assumed to hold, we take $\xi_{7}, \xi_{8}$, and $\xi_{9}$, so that

$$
\left(S_{\min }-\frac{C_{3}^{b d} V_{\max }^{2}}{A_{\min }}-\xi_{7}-\frac{C_{3}^{b d} \xi_{8}}{A_{\min }}\left(\frac{1}{\beta_{\min }}+1\right)-\frac{C_{1} \xi_{9} V_{\max }^{2}}{A_{\min }^{2}}\right)>\delta
$$

for some $\delta>0$, and taking $C_{4}^{b d}$ to be the global constant bound for (54) we obtain

$$
k^{2}\|u\|_{L^{2}(\Omega)}^{2} \leq \frac{C_{4}^{b d}}{\delta}\left(1+\frac{1}{k^{2}}\right)\left(\|f\|_{L^{2}(\Omega)}^{2}+\|g\|_{L^{2}\left(\Gamma_{R}\right)}^{2}\right),
$$

and using (41), and taking $C_{5}^{b d}$ to be the global constant bound we obtain

$$
\|\nabla u\|_{L^{2}(\Omega)}^{2} \leq C_{5}^{b d}\left(1+\frac{1}{k^{2}}\right)\left(\|f\|_{L^{2}(\Omega)}^{2}+\|g\|_{L^{2}\left(\Gamma_{R}\right)}^{2}\right),
$$

as desired. 


\section{References}

1. I. M. Babuska and S. A. Sauter, Is the pollution effect of the FEM avoidable for the Helmholtz equation considering high wave numbers?, SIAM Rev. 42 (2000), no. 3, 451-484.

2. T. Betcke, S. N. Chandler-Wilde, I. G. Graham, S. Langdon, and M. Lindner, Condition number estimates for combined potential integral operators in acoustics and their boundary element discretisation, Numer. Methods Partial Differential Equations 27 (2011), no. 1, 31-69. MR 2743599 (2012a:65355)

3. P. Cummings and X. Feng, Sharp regularity coefficient estimates for complex-valued acoustic and elastic Helmholtz equations, Math. Models Methods Appl. Sci. 16 (2006), no. 1, 139-160. MR 2194984 (2007d:35030)

4. D. A. Di Pietro and A. Ern, Mathematical aspects of discontinuous Galerkin methods, Mathématiques \& Applications (Berlin), vol. 69, Springer, Heidelberg, 2012. MR 2882148

5. S. Esterhazy and J. M. Melenk, On stability of discretizations of the Helmholtz equation, Numerical analysis of multiscale problems, Lect. Notes Comput. Sci. Eng., vol. 83, Springer, Heidelberg, 2012, pp. 285-324. MR 3050917

6. D. Gallistl and D. Peterseim, Stable multiscale Petrov-Galerkin finite element method for high frequency acoustic scattering, Comp. Meth. Appl. Mech. Eng. 295 (2015), $1-17$.

7. P. Grisvard, Contrôlabilité exacte des solutions de l'équation des ondes en présence de singularités, J. Math. Pures Appl. (9) 68 (1989), no. 2, 215-259. MR 1010769 (90i:49045)

8. P. Henning, P. Morgenstern, and D. Peterseim, Multiscale Partition of Unity, Meshfree Methods for Partial Differential Equations VII (M. Griebel and M. A. Schweitzer, eds.), Lecture Notes in Computational Science and Engineering, vol. 100, Springer, 2014.

9. U. Hetmaniuk, Stability estimates for a class of Helmholtz problems, Commun. Math. Sci. 5 (2007), no. 3, 665-678. MR 2352336 (2008m:35050)

10. Ch. Makridakis, F. Ihlenburg, and I. Babuška, Analysis and finite element methods for a fluid-solid interaction problem in one dimension, Mathematical Models and Methods in Applied Sciences 06 (1996), no. 08, 1119-1141.

11. J. M. Melenk, On generalized finite-element methods, ProQuest LLC, Ann Arbor, MI, 1995, Thesis (Ph.D.)-University of Maryland, College Park. MR 2692949

12. J. M. Melenk and S. A. Sauter, Wave-number explicit convergence analysis for Galerkin discretizations of the Helmholtz equation, SIAM J. Numer. Anal. 49 (2011), 1210-1243.

13. D. Peterseim, Eliminating the pollution effect in Helmholtz problems by local subscale correction, ArXiv e-prints 1411.1944 (2014).

14. H. Wu, Pre-asymptotic error analysis of CIP-FEM and FEM for the Helmholtz equation with high wave number. Part I: linear version, IMA J. Numer. Anal. 34 (2014), no. 3, 1266-1288. MR 3232452 